\title{
Fuel consumption and collision avoidance strategy in multi-static orbit formations
}

\author{
E.F. Jochim* , H. Fiedler, G. Krieger \\ Microwaves and Radar Institute, German Aerospace Center (DLR), Oberpfaffenhofen, Germany
}

\section{A R T I C L E I N F O}

\section{Article history:}

Received 26 February 2010

Received in revised form

17 August 2010

Accepted 16 September 2010

Available online 16 October 2010

Keywords:

SAR interferometry

Multi-static orbit formations

Relative satellite motion

Satellite manoeuvre costs

Cartwheel

Pendulum satellite formations

\begin{abstract}
A B S T R A C T
This paper analyses the fuel consumption of interferometric radar missions employing small satellite formations like, e.g., Cross-track Pendulum, Cartwheel, CarPe, or Trinodal Pendulum. Individual analytic expressions are provided for each of the following contributions: separation from a simultaneously injected master satellite, formation setup, orbit maintenance, formation maintenance, and distance maintenance. For this, a general system of equations is derived describing the relative motion of the small satellites in a co-rotating reference frame. The transformation into Keplerian elements is carried out. To evaluate fuel consumption, three master satellites are assumed in different orbital heights, which are typical for Earth observation missions. The size of the exemplarily analysed formations is defined by remote sensing aspects and their respective fuel requirements are estimated. Furthermore, a collision avoidance concept is introduced, which includes a formation separation and formation set-up after a desired time period.
\end{abstract}

(c) 2010 Elsevier Ltd. All rights reserved.

\section{Introduction}

One major topic in Earth observation is the derivation of a highly accurate digital elevation model (DEM) on a global scale $[7,18,22]$. Spaceborne synthetic aperture radar (SAR) interferometry is a powerful and well-established remote sensing technique that allows for the derivation of global DEMs in short time periods with an excellent cost-benefit ratio [15]. The generation of high-quality interferograms requires the acquisition of two or more SAR images from slightly different orbital positions (i.e. the satellites are separated by different so-called baselines), if possible simultaneously. This can be achieved with a constellation or formation of receive-only satellites following a fully active illuminating satellite [17,8,23]. Several formations like, e.g. Cartwheel or Cross-Track Pendulum have been proposed $[10,11,17,19,21]$ and analysed in the last $\sim 10$ years mostly with respect to SAR performance [14].

\footnotetext{
* Corresponding author. Tel.: +49815328 2738; fax: +49815328 1452 .

E-mail addresses: Fritz.Jochim@dlr.de, fritz.jochim@gmx.de (E.F. Jochim).
}

In this paper, we analyse such constellations with respect to fuel consumption. Therefore, a general description of the motion of the small satellites is derived first and its transformation into Keplerian elements. It is assumed that the small satellites are injected into their orbits together with the master satellite using the same launcher. First, the satellites of the formation are separated from the master satellite (here denoted as distance acquisition) and then the formation is set up (formation acquisition). Now, the formations are analysed with respect to formation maintenance and orbit maintenance (including distance maintenance). The latter is important to avoid a collision between the master (illuminator) satellite and the formation. The term 'formation' refers to the small satellites and the term 'master satellite' to the illuminating satellite.

\section{Microsatellite orbit parameter evaluation}

For performing synthetic aperture radar (SAR) interferometry with small microsatellites there were two proposals in 1998: Techsat 21 [5,20] and Cartwheel [17]. Techsat 21 is based on a close formation of three fully 
active SAR satellites. In contrast, the Cartwheel formation uses a separate master satellite for radar signal transmission. The ground reflected signal is then simultaneously recorded by three low-cost passive receiver satellites. The averaged orbit of the master satellite is circular, whereas the microsatellites have small deviations in their respective Keplerian elements to build up their specific formation. By changing the Keplerian elements, new members for such formations joined in the meantime the family of formation flying satellites, e.g. the Pendulum or CarPe and the HELIX [14], the latter will be operationally flown first by DLR [16]. The basic features of the constellations used in the present paper are shortly described in the following section.

In the frame of his considerations of the three body problem, Hill [9] investigated the relative motion of small bodies in relation to a centre body taking into account simplified assumptions. The centre body is assumed with no mass. Its orbit around the primary (here the Earth) should be an unperturbed circular path. The orbits of the second satellite (here microsatellite) should deviate from the orbit of the master satellite by small modifications only. In order to get a feeling of the relative motion of the microsatellite, an unperturbed Keplerian orbit will be assumed. Hill derived the equations of motion of this relative motion (denoted as "Hill's equations", later rediscovered by the name "Clohessy-Wiltshire equations" [3]) and found a solution of these equations by integration with respect to the assumptions mentioned earlier.

However, it is not necessary to undertake the integration within the frame of an investigation of the relative motion. The reason is simply the fact that both orbits are known by definition. Therefore, it will only be necessary to find the relative motion of the microsatellite in a corotating co-ordinate system of the master satellite. By this way, it will be possible to find a relation of the relative motion of the microsatellite represented by modifications of the Keplerian parameters of the master satellite. This will allow for a parametrization of the relative motion, which will ease the estimation of fuel requirements.

The co-rotating system of the centre body is defined by the radial direction vector $\vec{r}_{M 0}=: \vec{q}_{1}$, the transversal direction vector $\vec{q}_{M 0}=: \vec{q}_{2}$, and the normal direction vector $\vec{r}_{C 0} \times \vec{q}_{C 0}=: \vec{q}_{3}$ with $C$ indicating the centre body and $\vec{r}$ being the radius vector. The equatorial basic system is defined by the orthogonal direction vectors $\vec{p}_{i}$, so a transformation from the basic system into the co-rotating system is given by $\vec{p}_{i}=a_{C i}^{j} \vec{q}_{j}$. Here, the index $j$ denotes the Einstein summation convention. The transformation matrix $a_{C i}^{j}$ is well defined by the Keplerian inclination $i$, the right ascension of the ascending node $\Omega$, and argument of latitude $u$. The mean radius of the centre body is $r_{C}=$ const., its mean eccentricity $e_{C}=0$. Its other Keplerian elements are also assumed to be constant. Therefore, with

$$
\begin{aligned}
& \vec{r}_{C}=r_{C} \vec{r}_{C 0}=r_{C} a_{C 1}^{i} \vec{p}_{i}=r_{C} a_{C 1}^{i} a_{C i}^{j} \vec{q}_{j} \\
& \text { with } a_{C 1}^{i} a_{C i}^{1}=1 \text { and } a_{C 1}^{i} a_{C i}^{j}=0 \text { for } j=2,3
\end{aligned}
$$

the microsatellite will get the radius vector

$$
\vec{r}_{S}=r_{S} a_{S 1}^{i} \vec{p}_{i}=r_{S} a_{S 1}^{i} a_{C i}^{j} \vec{q}_{j}
$$

Its Keplerian elements contained in the transformation matrix $a_{S 1}^{i}$ will show slight deviations of the elements of the centre body. The relative position vector of the microsatellite with respect to the co-rotating system of the centre body will be

$\Delta \vec{r}=\vec{r}_{S}-\vec{r}_{C}=r_{S} a_{S 1}^{i} a_{C i}^{j} \vec{q}_{j}-r_{C} \vec{q}_{1}$

The coefficients of the transformation matrix of the microsatellite are related to the centre body by the relations

$$
\begin{aligned}
& a_{S}=r_{C} \\
& e_{S}=\Delta e, \quad \Delta e \ll 1 \\
& i_{S}=i_{C}+\Delta i, \quad \Delta i \ll 1^{\circ} \\
& \boldsymbol{\Omega}_{S}=\boldsymbol{\Omega}_{C}+\Delta \boldsymbol{\Omega}, \quad \Delta \boldsymbol{\Omega} \ll 1^{\circ} \\
& u_{S}=v_{S}+\omega_{S}=u_{C}+\Delta u, \quad \Delta u \ll 1^{\circ}
\end{aligned}
$$

where $v_{S}$ denotes the true anomaly of the microsatellite and $\omega_{s}$ is the argument of perigee. These values set into the transformation matrix will lead to

$a_{S i j}=a_{C i j}+\Delta a_{C i j}$

with the approximations

$\Delta a_{C 11} \approx \Delta i a_{C 31} \sin \left(u_{C}\right)+\Delta u a_{C 21}-\Delta \boldsymbol{\Omega} a_{C 12}$

$\Delta a_{C 12} \approx \Delta i a_{C 32} \sin \left(u_{C}\right)+\Delta u a_{C 22}+\Delta \boldsymbol{\Omega} a_{C 11}$

$\Delta a_{C 13} \approx \Delta i a_{C 33} \sin \left(u_{C}\right)+\Delta u a_{C 23}$

$\Delta a_{C 21} \approx \Delta i a_{C 31} \cos \left(u_{C}\right)-\Delta u a_{C 11}-\Delta \boldsymbol{\Omega} a_{C 22}$

$\Delta a_{\mathrm{C} 22} \approx \Delta i a_{\mathrm{C} 32} \cos \left(u_{\mathrm{C}}\right)-\Delta u a_{\mathrm{C} 12}+\Delta \boldsymbol{\Omega} a_{\mathrm{C} 21}$

$\Delta a_{C 23} \approx \Delta i a_{C 33} \cos \left(u_{C}\right)-\Delta u a_{C 13}$

$\Delta a_{\mathrm{C} 31} \approx \Delta i a_{\mathrm{C} 33} \sin \left(\boldsymbol{\Omega}_{\mathrm{C}}\right)-\Delta \boldsymbol{\Omega} a_{\mathrm{C} 32}$

$\Delta a_{\mathrm{C} 32} \approx-\Delta i a_{\mathrm{C} 33} \cos \left(\boldsymbol{\Omega}_{\mathrm{C}}\right)+\Delta \boldsymbol{\Omega} a_{\mathrm{C} 31}$

$\Delta a_{C 33} \approx-\Delta i \sin \left(i_{C}\right)$

Because of the small eccentricity $e_{S}$, the radius of the microsatellite can be linearised

$r_{S}=\frac{a_{S}\left(1-e_{S}^{2}\right)}{1+e_{S} \cos \left(v_{S}\right)} \approx r_{M}\left(1-\Delta e \cos \left(v_{S}\right)\right)$,

where $a_{S}$ is the semi-major axis of the microsatellite. In order to guarantee the identical Keplerian period for both satellites, $a_{S}=r_{S}$ has to be assumed. Then

$$
\begin{aligned}
\Delta \vec{r}= & x \vec{q}_{1}+y \vec{q}_{2}+z \vec{q}_{3} \\
& \approx r_{C}\left(\Delta a_{C 1}^{i} a_{C i}^{j}-\Delta e \cos \left(v_{S}\right) a_{C 1}^{i} a_{C i}^{j}\right) \vec{q}_{j} .
\end{aligned}
$$

Introducing the coefficients $a_{C i}^{j}$ of the transformation matrix of the centre body including the condition in Eq. (1) the coefficients of the relative position vector of the microsatellite in the co-rotating system of the centre body will be

$$
\begin{aligned}
& x=-r_{C} \Delta e \cos \left(v_{S}\right) \\
& y=r_{C}\left(\Delta \boldsymbol{\Omega} \cos \left(i_{C}\right)+\Delta u\right) \\
& z=r_{C}\left(\Delta i \sin \left(u_{C}\right)-\Delta \boldsymbol{\Omega} \sin \left(i_{C}\right) \cos \left(u_{C}\right)\right)
\end{aligned}
$$

The true anomaly $v_{S}$ will be approximated by the first two terms of the equation of centre

$v_{S}=M_{S}+2 \Delta e \sin \left(M_{S}\right)+\cdots$

Then

$\cos \left(v_{S}\right) \approx \cos \left(M_{S}\right)-2 \Delta e \sin ^{2}\left(M_{S}\right)+\cdots$

and it will remain for the first coefficient of the relative position vector

$x=-r_{C} \Delta e \cos \left(M_{S}\right)+\cdots$ 
The mean anomaly of the microsatellite differs from the mean anomaly of the centre body by $\Delta M$, which must be sufficient to ensure safe operation

$M_{S}=M_{C}+\Delta M$

The mean anomaly will be computed from

$M_{C}=M_{C 0}+n_{C}\left(t-t_{0}\right)$

with $n_{C}$ being the mean Keplerian motion of the centre body. The first coefficient will get the form (the argument $270^{\circ}$ was chosen in order to eliminate the negative sign in the equation)

$x=r_{C} \Delta e \sin \left[M_{C 0}+\Delta M+270^{\circ}+n_{C}\left(t-t_{0}\right)\right]+\cdots$

and the second coefficient will be

$$
\begin{aligned}
y= & r_{C}\left\{\Delta \boldsymbol{\Omega} \cos \left(i_{C}\right)+\Delta M+\Delta \omega+2 \Delta e \cos \left[M_{C 0}+\Delta M+270^{\circ}\right.\right. \\
& \left.\left.+n_{C}\left(t-t_{0}\right)\right]\right\}+\cdots
\end{aligned}
$$

By applying the following with abbreviations with $r_{C}=a_{C}$,

$A_{i}=a_{C} \Delta e_{i}$

$\alpha_{i}=M_{C 0}+\Delta M_{i}+270^{\circ}$

$\Delta y_{i}=a_{C}\left(\Delta M_{i}+\Delta \omega+\Delta \boldsymbol{\Omega}_{i} \cos \left(i_{C}\right)\right)$

$u_{C 0}=M_{C 0}+\omega_{C}$

$B_{i} \sin \left(\beta_{i}\right)=a_{C}\left[\Delta i_{i} \sin \left(u_{C 0}\right)-\Delta \boldsymbol{\Omega}_{i} \sin \left(i_{C}\right) \cos \left(u_{C 0}\right)\right]$

$B_{i} \cos \left(\beta_{i}\right)=a_{C}\left[\Delta i_{i} \cos \left(u_{C 0}\right)+\Delta \boldsymbol{\Omega}_{i} \sin \left(i_{C}\right) \sin \left(u_{C 0}\right)\right]$

to these equations, one will obtain the usual form of the relative position vector

$x_{i}=A_{i} \sin \left[n_{C}\left(t-t_{0}\right)+\alpha_{i}\right]+\cdots$

$y_{i}=2 A_{i} \cos \left[n_{C}\left(t-t_{0}\right)+\alpha_{i}\right]+\Delta y_{i}+\cdots$

$z_{i}=B_{i} \sin \left[n_{C}\left(t-t_{0}\right)+\beta_{i}\right]+\cdots$

In these equations the index $i$ refers to the $i$ th satellite of a formation as considered in the following sections of this paper. It may be assumed that the master satellite will be set at the origin of the above defined co-rotating co-ordinate system with a well-defined offset in along track. The Keplerian elements of the master satellite are known as well as the relative Keplerian elements of the microsatellites. On the other hand, from the parameters of the relative motion the Keplerian elements of the microsatellites will be obtained, which is one great advantage of the above derivation. From expressions (17) follows

$$
\begin{aligned}
& \Delta e_{i}=\frac{A_{i}}{a_{M}} \\
& \Delta M_{i}=\alpha_{i}-M_{M 0}-270^{\circ} \\
& u_{M 0}=M_{M 0}+\omega_{M} \\
& \Delta i_{i}=\frac{B_{i}}{a_{M}} \cos \left(u_{M 0}-\beta_{i}\right) \\
& \Delta \boldsymbol{\Omega}_{i} \sin \left(i_{M}\right)=\frac{B_{i}}{a_{M}} \sin \left(u_{M 0}-\beta_{i}\right) \\
& \Delta \omega_{i}=\frac{\Delta y_{i}}{a_{M}}-\Delta M_{i}-\Delta \boldsymbol{\Omega}_{i} \cos \left(i_{M}\right) .
\end{aligned}
$$

As seen from these formulae, the so-called inclination and eccentricity vectors are not required in the case of remote sensing satellites as considered in this paper for any inclination with $\sin (i) \neq 0$.

\section{Satellite parameters and formations}

For the following analyses, three different heights of the master satellite above the Earth's surface are assumed: 500,650 , and $800 \mathrm{~km}$. This covers roughly the range in which most of the Earth remote sensing satellites are operated. For demonstrating effects of the master satellite's mass, two values are assumed: 1000 and $4000 \mathrm{~kg}$. The cross sections analysed are $2 \mathrm{~m}^{2}$ (e.g. TerraSAR-X like satellite) and $20 \mathrm{~m}^{2}$ (e.g. DAICHI like satellite, formerly known as ALOS).

\subsection{Microsatellites}

All single microsatellites are assumed with a satellite mass of $130 \mathrm{~kg}$ and a cross section of $2 \mathrm{~m}^{2}$. It is assumed that any formation will orbit at almost the same semimajor axis, inclination, and eccentricity like the master satellite. The orbits of the microsatellites will be described by the relative position vector presented in the formulae of Section 2. From Eq. (18) it is clearly visible that any motion along the $x$-axis implies a coupling of the $y$-axis,

\begin{tabular}{|c|c|c|c|c|c|}
\hline Name of formation & $A_{i}$ & $\alpha$ & $\boldsymbol{B}_{\boldsymbol{i}}$ & $\beta$ & $\Delta y_{i}$ \\
\hline Cartwheel & $A_{1}=A_{2}=A_{3}$ & $\alpha_{i}=i 120^{\circ}+\mathrm{k}$ & $B_{i}=0$ & $\beta_{i}=$ arbit. & $\Delta y_{i}=0$ \\
\hline Cross-track Pendulum & $A_{i}=0$ & $\alpha_{i}=$ arbit. & $B_{1}=B_{2}=B_{3}$ & $\beta_{i}=i 120^{\circ}+\mathrm{k}$ & $\Delta y_{i} \neq \Delta y_{j}$ with $i \neq j$ \\
\hline Trinodal Pendulum & $A_{i}=0$ & $\alpha_{i}=$ arbit. & $B_{i}=$ arbit. & $\begin{array}{l}\beta_{1}=90^{\circ} \text { or } 270^{\circ} ; \beta_{2}=0^{\circ} \\
\beta_{3}=90^{\circ} \text { or } 270^{\circ}\end{array}$ & $\Delta y_{i} \neq \Delta y_{j}$ with $i \neq j$ \\
\hline Trinodal Pendulum* & $\begin{array}{l}A_{1}=A_{3}=\text { small } \\
A_{2}=0\end{array}$ & $\alpha_{1}=90^{\circ}, \alpha_{2}=$ arbit., $\alpha_{3}=270^{\circ}$ & $B_{i}=$ arbit. & $\begin{array}{l}\beta_{1}=90^{\circ} \text { or } 270^{\circ} ; \beta_{2}=0^{\circ} \\
\beta_{3}=90^{\circ} \text { or } 270^{\circ}\end{array}$ & $\begin{array}{l}\Delta y_{1} \neq \Delta y_{3} \\
\Delta y_{2}=0 \\
\Delta y_{1,2} \neq 0\end{array}$ \\
\hline CarPe & $\begin{array}{l}A_{1}=A_{2}=0 ; A_{3}=\mathrm{k} \\
\sqrt{ }(3 / 2)\end{array}$ & $\alpha_{1,2}=$ arbit., $\alpha_{3}=0$ & $B_{1}=B_{2}=k \cdot \sqrt{ }(1 / 2) ; B_{3}=0$ & $\beta_{1}=90^{\circ} ; \beta_{2}=270^{\circ} ; \beta_{3}=$ arbit. & $\Delta y_{1} \neq \Delta y_{2} ; \Delta y_{i}=$ arbit. \\
\hline HELIX & $\begin{array}{l}A_{1}=0 \\
A_{2}=\text { arbit. }\end{array}$ & $\alpha_{1}=$ arbit.; $\alpha_{2}= \pm 180^{\circ}$ & $\begin{array}{l}B_{1}=0 \\
B_{2}=\text { arbit. }\end{array}$ & $\beta_{1}=$ arbit. $\beta_{2}=90^{\circ}$ & $\Delta y_{1}=$ arbit. $\Delta y_{2}=$ arbit. \\
\hline TechSAT 21 & $A_{1}=A_{2}=A_{3}$ & $\alpha_{i}=i 120^{\circ}+k$ & $A_{i}=B_{i}$ & $\beta_{i}=\alpha_{i}$ & $\Delta y_{i}=0$ \\
\hline
\end{tabular}
while the horizontal cross-track motion of the satellite is completely decoupled. In literature, many formations may

Table 1

Example of formations and their respective parameters as formulated in (18). The Trinodal Pendulum* is the same as the Trinodal Pendulum, but with additional small eccentricity offsets to avoid a collision between the satellites. In the HELIX formation it is assumed that the first satellite is placed at the centre of the co-rotating co-ordinate system; another solution would be a symmetrically distribution of the two satellite around the centre. In this table, $k$ means a freely selectable offset or constant, non-zero for the CarPe case. 


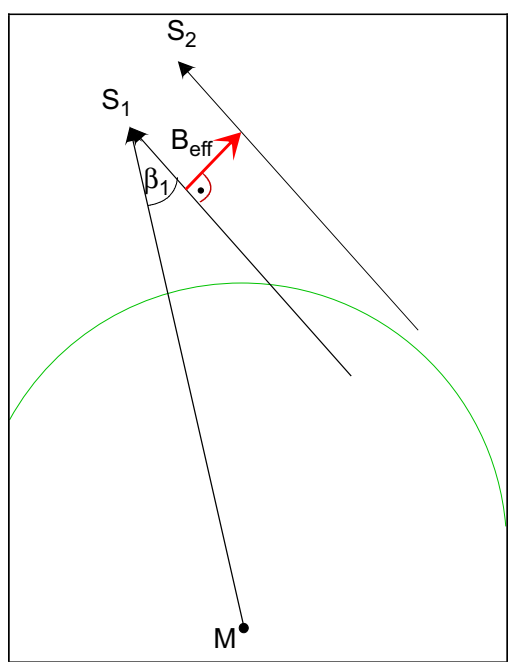

Fig. 1. The effective baseline $B_{\text {eff }}$ for cross-track interferometry between the two satellite positions $S_{1}$ and $S_{2}$. The lines of sight as seen from satellite positions $S_{1}$ and $S_{2}$ are assumed to be parallel in case of small distances between the satellites.

be described by these formulae and their names and parameters are given in Table $1,[6,10,14,16,17]$. Note that all the formations described in this Table consist in this specific case of three satellites (exception: Helix consisting of two satellites), which of course can be adapted to more satellites for specific mission goals.

The value of the parameters will be triggered by the size of the formation, i.e. the relative positions between the satellites, which may be defined by the effective baseline $B_{\text {eff. }}$. This effective baseline (cf. Fig. 1) must always be considered in conjunction with the radar wavelength, and assumed lengths in the order of several kilometres for interferometric observations in L-Band.

From Table 1 , it is easy to calculate the required Keplerian elements of the microsatellites and their respective deviations from the master satellite using formulae (19). To build up any formation based on these deviations, fuel and manoeuvres will be required. This will be derived in the next Section.

\section{Orbit mechanical background}

The analysis of the fuel consumption is split into five parts: separation of a formation of satellites from the master satellite immediately after injection, set-up of the formation, orbit maintenance, formation maintenance, and distance maintenance. The formation shall orbit in front of or behind the master satellite within a certain distance range. Keeping the formation within this range is denoted distance maintenance. As the master satellite will be independently controlled, this motion has to be analysed first. Then, the distance maintenance of the formation can be evaluated. For the fuel consumption of formation maintenance, the formations have to be analysed individually. The fuel mass $M_{T}$ required to allow for the velocity increment of a rocket or satellite with initial mass $M_{0}$ will be calculated from

$M_{T}=M_{0}\left(1-e^{-\left(\Delta V / c_{e}\right)}\right)$

with the velocity increment $\Delta V$ and the exhaust velocity $c_{e}$. In the following, an exhaust velocity of $c_{e}=2800 \mathrm{~m} / \mathrm{s}$ is assumed for the on-board liquid hot gas thrusters' systems.

The analytical investigation includes secular secondorder values of the first three zonal coefficients $J_{2}, J_{3}$, and $J_{4}$ of the Earth's gravitational field, first-order periodic variations due to $J_{2}$, secular variations due to air drag with respect to maximum and minimum solar flux estimations, secular variations in inclination due to Sun and Moon averaged attraction. Minor effects like, e.g. different solar radiation effects due to the shadowing of the Earth are not considered.

The mathematical formulae applied for the estimation of the required velocity increments are presented in the following sections.

\subsection{Separation between the formation and the master satellite}

There are three different possibilities for separating the three satellites from the simultaneously injected master satellite: separation based on anomalistic motion [12], separation based on very small velocity increments [13], and separation based on changes in semi-major axis.

\subsubsection{Orbit position separation based on anomalistic motion}

The separation of two satellites moving on the same position in the same orbit can be achieved by the following strategy (as indicated in Fig. 2): both satellites are moving on a (near) circular orbit with mean semi-major axis $\bar{a}_{1}$ and mean anomalistic period $\bar{P}_{1 a}=2 \pi / \bar{n}_{1 a}$ with mean anomalistic mean motion $\bar{n}_{1 a}=\bar{n}_{10}+\dot{M}_{10 \text { s }}$, where $\bar{n}_{10}=\sqrt{\mu / \bar{a}_{1}}$ is the Keplerian mean motion, $\mu=398600.4415 \times 10^{9} \mathrm{~m}^{3} / \mathrm{s}^{2}$ being the geocentric gravitational constant, and $\dot{M}_{10 \text { s }}$ the secular drift of the mean epoch anomaly. After the

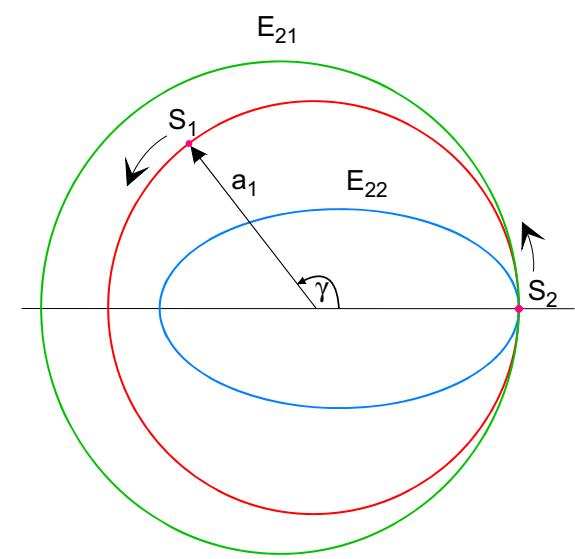

Fig. 2. Two satellites separated by the angle $\gamma$ will be put into a close configuration (or vice versa) by a manoeuvre of the second satellite in the position $S_{2}$ by means of an elliptical transfer orbit ( $E_{21}$ outside, $E_{22}$ inside the orbit of the master satellite $S_{1}$ with radius $a_{1}$ ). The procedure demonstrated in this figure is sometimes also known as "phasing orbit". 
manoeuvre the satellites should be separated along the same orbit by the central angle $\gamma$. In position $S_{2}$, the second satellite will be injected into an elliptic orbit with mean semi-major axis $\bar{a}_{2}$ and mean eccentricity $\bar{e}_{2}$ and mean anomalistic period $\bar{P}_{2 a}=2 \pi / \bar{n}_{2 a}$, so that $N$ anomalistic periods $\bar{P}_{1 a}$ are matching $M$ anomalistic periods $\bar{P}_{2 a}$ :

$\bar{P}_{a 2}=\frac{1}{M}\left(N \bar{P}_{a 1}+\sigma_{e} \Delta t\right)$

with $\sigma_{e}$ positioning the second satellite in front of the prime satellite $\left(\sigma_{e}=-1\right)$ or behind $\left(\sigma_{e}=+1\right)$. Assuming a circular motion of the uninfluenced satellite with its circular velocity $V_{c}$ the time interval will be calculated to be

$\Delta t=\frac{\gamma}{n_{1 a}} \approx \frac{\Delta x}{V_{c}}$

where $\gamma$ is the separation angle and $\Delta x$ the distance of position change along the orbit.

As a second condition the perigee/apogee radius will be used in the form

$\bar{r}_{1}=\bar{a}_{2}\left(1+\sigma_{e} \bar{e}_{2}\right)=\bar{a}_{1} \quad$ and $\quad \sigma_{\mathrm{e}}=\left\{\begin{array}{cc}+1 & \text { if } \bar{P}_{a 2}>\bar{P}_{a 1} \\ -1 & \text { if } \bar{P}_{a 2}<\bar{P}_{a 1}\end{array}\right.$

From the last two equations, $\bar{a}_{2}$ and $\bar{e}_{2}$ will be calculated by an iteration process. Achieving position $S_{1}$ on the first orbit, a manoeuvre with same size as in position $S_{2}$ but in opposite direction has to be performed in order to reach orbit 1 in $S_{1}$. This manoeuvre will be calculated by the difference in velocity between orbit 1 and orbit 2: in the apogee of orbit 2 if $\bar{P}_{a 2}<\bar{P}_{a 1}$, and in the perigee of orbit 2 if $\bar{P}_{a 2}>\bar{P}_{a 1}$. The velocity on orbit 2 in the moment of the manoeuvre will be

$V_{2}=\sqrt{\frac{\mu}{\bar{a}_{1}}\left(1+\sigma_{e} \bar{e}_{2}\right)}$

Finally the amount in velocity increment in tangential direction for the two required manoeuvres will be

$\Delta V=2\left(V_{2}-V_{1}\right) \quad$, where $V_{1}=\sqrt{\frac{\mu}{a}}$.

If $\Delta V<0$, the manoeuvre takes place in the apogee of orbit 2 and the impulse has to be directed opposite to the flight direction of the satellite on its orbit.

A tradeoff between time span and required fuel consumption can be found in systematic tables varying the integer numbers $N$ and $M$ [12]. As an example, a shift of the second satellite by $\Delta x=50 \mathrm{~km}$ behind the prime satellite after 61 anomalistic periods of both satellites should be achieved in the TerraSAR-X case (nominal orbital data: sunsynchronous orbit, reproducible after 11 days and 167 draconic periods, semi-major axis $a_{1}=6883.507 \mathrm{~km}$, mean eccentricity $e_{1}=0.0$, inclination $i_{1}=97.445789^{\circ}$, circular velocity $V_{1}=7.6096 \mathrm{~km} / \mathrm{s}$ ). The anomalistic period for the first satellite is $P_{a 1}=94.790 \mathrm{~min}$ and for the second satellite, $P_{a 2}=94.788 \mathrm{~min}$. The transfer orbit will have a semi-major axis of $a_{2}=6883.420 \mathrm{~km}$ with an eccentricity of $e_{2}=0.0000126$. The complete transfer time will last $t=96.367841 \mathrm{~h}$ with a velocity increment (2-impulse procedure) of $\Delta V=0.09585 \mathrm{~m} / \mathrm{s}$, corresponding to a fuel mass of $4.45 \mathrm{~g}$ for the small satellite with $m=130 \mathrm{~kg}$ and a hot gas engine $\left(c_{e}=2800 \mathrm{~m} / \mathrm{s}\right)$.

\subsubsection{Orbit position separation using very small velocity increments}

For estimations of small orbital variations the third Keplerian law will be used in the classical form $P=2 \pi / n=2 \pi\left(a^{3} / \mu\right)^{1 / 2}=2 \pi a / V_{c}$, where $n$ is the Keplerian mean motion and $V_{C}$ the circular velocity. Then a small variation in the semi-major axis will cause the variation in Keplerian period $\Delta P \approx 3 \pi \Delta a / V_{C}$. For small orbital changes caused by a velocity increment applied in transversal direction using the formula

$\Delta a=\dot{a}_{s} \Delta t$

the semi-major axis will be changed by [13]

$\Delta a \approx-\frac{2 a}{V_{C}} \Delta V_{T}=-\frac{P}{\pi} \Delta V_{T}$.

Therefore a relation between the variation in period corresponds to a variation in transversal direction $\Delta P \approx-3 P \Delta V_{T} / V_{C}$. The deviation $\Delta x$ along a circular orbit in the time interval $\Delta t$ can be calculated from $\Delta x=V_{C} \Delta t$. If the time interval is assumed a Keplerian period, the shift along the orbit can be calculated from

$\Delta x_{P}=V_{c} \Delta P \approx-\frac{6 a \pi}{V_{c}} \Delta V_{T}$

Considering the relative motion of a changed orbit by the velocity increment $\Delta V_{T}$ with respect to an unchanged orbit, the relative time interval $\Delta t_{r}$ corresponding to a relative drift $\Delta x_{r}$ holds the relation $\Delta x_{P} / P=\Delta x_{r} / \Delta t_{r}$. This leads to the orbital shift within the time interval $\Delta t_{r}$ caused by the velocity increment $\Delta V_{T}$

$\Delta x_{r} \approx-3 \Delta t_{r} \Delta V_{T}$

From this estimation the time interval needed for the relative transfer of the length $\Delta x_{r}$ caused by the velocity increment $\Delta V_{T}$ can be estimated

$\Delta t_{r} \approx-\frac{\Delta x_{r}}{3 \Delta V_{T}}$

However, in this case three small manoeuvres will be required in order to perform the location separation: at the beginning in order to get a slightly modified orbit of the master orbit with respect to the unchanged master orbit, a midterm manoeuvre in order to change the flight direction and to go back to the master orbit, and a stop manoeuvre to fix the satellite on the master orbit in the required distance $\Delta x$ from the master satellite are required. This is shown in Fig. 3.

As an example, for the same manoeuvre as described in Section 4.1 .1 with $\Delta x=50 \mathrm{~km}$ and a transfer time of $t=96.367841 \mathrm{~h}$, neglecting the midterm manoeuvre, the velocity increment for the two manoeuvres 1 and 3 will be together $\Delta V_{T}=0.096082 \mathrm{~m} / \mathrm{s}$ corresponding to a fuel consumption of $4.46 \mathrm{~g}$ for a $130 \mathrm{~kg}$ small satellite.

\subsubsection{Orbit position separation using any changes in semi- major axis}

In order to estimate a small relative shift of a satellite along its orbit by a true anomaly interval $\Delta v$ a circular orbit will be assumed. In this case the true anomaly interval will be replaced by the corresponding interval 
in mean anomaly $\Delta v \approx \Delta M=M_{i}-M,(i=1,2)$ with $M=M_{0}+n$ $\left(t-t_{0}\right), M_{i}=M_{0}+n_{i}\left(t_{i}-t_{0}\right)$.

As presented in Fig. 4 the following procedure takes place: let $a$ be the semi-major axis of the master orbit and $n$ the (Keplerian) mean motion along this orbit. A small change $\Delta a$ in semi-major axis using a tangential manoeuvre according to formula (26) will put the satellite after circularization (via 2 impulses) to an orbit with a slightly different semi-major axis $a_{1 / 2}=a \pm \Delta a$, where the higher orbit is chosen to get a location behind the master position, the lower orbit to get a location before the master. The mean motion of the second orbit will be $n_{i}=\left(\mu / a_{i}^{3}\right)^{1 / 2}$. Starting with the same mean anomaly $M_{0}$ after a time interval $\Delta t=t-t_{0}$ along the two orbits the mean anomalies $M$ and $M_{i}$ will be reached. The desired position difference $\Delta v$ between the master and the second

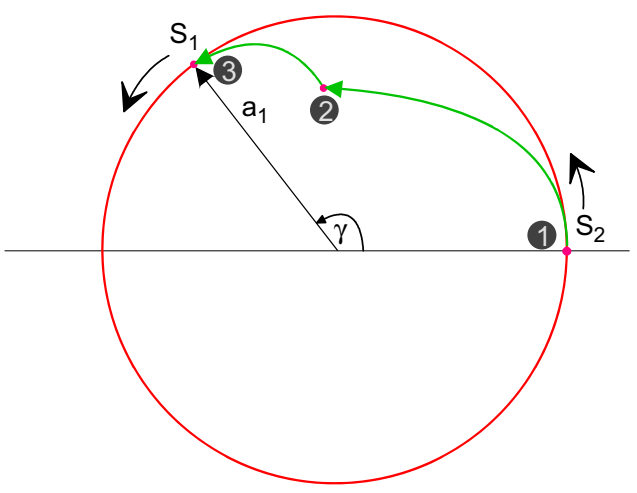

Fig. 3. Two satellites separated by the relative angle $\gamma$ will be put into the requested relative position by three small manoeuvres along two elliptic orbital arcs: way from position $S_{2}$ to position $S_{1}$ or vice versa.

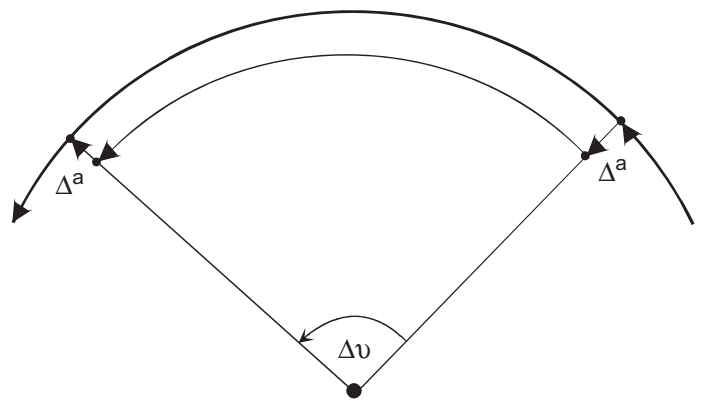

Fig. 4. Small position shift along an orbit with four impulses. position corresponds to

$\Delta v \approx \Delta M=M_{i}-M=\left(n_{i}-n\right)\left(t_{i}-t_{0}\right)=\left(n_{i}-n\right) \Delta t$

Therefore, after the time interval $\Delta t=\Delta v /\left|n_{i}-n\right|$ the requested position will be achieved. The second pair of manoeuvres will be executed in order to place the satellite back to the master orbit, however, by distance $\Delta v$ to the master. The time duration for the whole manoeuvre cycle depends on the velocity increments $\Delta V_{T}$ in order to change the semi-major axis by the value $\Delta a$ (according to formula (26)). On the other hand, if a change $\Delta a$ in semi-major axis is selected, the corresponding velocity increment will be calculated and the time interval $\Delta t$ will be obtained. A factor 10 in $\Delta a$ will lead approximately to a factor $10^{-1}$ in time.

Again, with the example parameters of $\Delta x=50 \mathrm{~km}$ and the transfer time of $t=96.367841 \mathrm{~h}$, the corresponding angle will be $\Delta v=0.4161816^{\circ}$ and the Keplerian mean motion $n=1.105489 \times 10^{-3} / \mathrm{s}$. The semi-major axis will be $6883.420087 \mathrm{~km}$ with a change of $\Delta a=-0.086913 \mathrm{~km}$. With the formulae mentioned earlier we obtain a velocity increment of $\Delta V=0.096 \mathrm{~m} / \mathrm{s}$, corresponding to a fuel mass of $4.46 \mathrm{~g}$.

Note: as seen from the examples mentioned earlier, the method described in Section 4.1.1 is a slightly less fuel demanding solution and as an asset it is generally applicable, while the other two methods are approximations. Furthermore, the first method requires two impulses while the 'small velocity increment' (Section 4.1.2) method requires three impulses and the semi-major axis change (Section 4.1.3), four impulses.

\subsection{Set-up of formation}

For the set-up of the formations, different manoeuvres must be executed: ascending node separation, inclination separation, eccentricity change, and change in the argument of perigee. For each formation the demands are different and summed up in Table 2.

\subsubsection{Semi-major axis manoeuvre}

The velocity increment needed for the changes in semi-major axis $a$ with value $\Delta a$ can be computed with sufficient accuracy for small changes from the rough formula

$\Delta V=\frac{V_{C}}{2 a} \Delta a$

Table 2

Kind of manoeuvre depending on formation for formation set-up (S) and for formation maintenance (M). Note, for the Trinodal Pendulum forces onto the ascending node are the same for all satellites and are negligible.

\begin{tabular}{llll}
\hline Manoeuvre & Cross-Track Pendulum & Cartwheel & CarPe \\
\hline Ascending Node & S, M & & S, M \\
Inclination & S, M & S & S, M \\
Arg. Perigee & & S & S, M \\
Eccentricity & M & M & M \\
Semi-major axis & S, M & S, M & S \\
Anomaly & & S & M \\
\hline
\end{tabular}


with the circular velocity $V_{C}$. All fuel masses in this paper are calculated according to (20).

\subsubsection{Eccentricity manoeuvre}

For near circular orbits the velocity increment

$\Delta V_{T}= \pm \frac{V_{C}}{2} \Delta e$

will be required to change the eccentricity by a value of $\Delta e$. The thrust has to be applied in tangential direction at the perigee or apogee of the orbit. The eccentricity interval will be obtained assuming a secular change in eccentricity from the expression $\Delta e=\dot{e}_{s} \Delta t$ with $\dot{e}_{S}$ being the secular change of the eccentricity.

\subsubsection{Inclination and ascending node manoeuvre}

The orbits of the Cross-Track Pendulum will be separated by different inclinations. The secular drift of the ascending node [1] is given by the equation

$$
\begin{aligned}
\dot{\boldsymbol{\Omega}}_{s G}= & -\frac{3}{2} \bar{n}_{0} J_{2} \frac{R_{E}^{2}}{\bar{p}_{0}^{2}} \cos \bar{i}_{0}+ \\
& +\frac{3}{2^{5}} \bar{n}_{0} \frac{R_{E}^{4}}{\bar{p}_{0}^{4}} \cos \bar{i}_{0}\left\{J _ { 2 } ^ { 2 } \left[-5+12 \sqrt{1-\bar{e}_{0}^{2}}+9\left(1-\bar{e}_{0}^{2}\right)\right.\right. \\
& \left.-\left(35+36 \sqrt{1-\bar{e}_{0}^{2}}+5\left(1-\bar{e}_{0}^{2}\right) \cos ^{2} \bar{i}_{0}\right)\right] \\
& \left.-5 J_{4}\left[5-3\left(1-\bar{e}_{0}^{2}\right)\right]\left(3-7 \cos ^{2} \bar{i}_{0}\right)\right\}+\ldots
\end{aligned}
$$

with the mean Keplerian motion $\bar{n}_{0}=\sqrt{\mu / \bar{a}_{0}{ }^{3}}$. The orbital elements used in this expression are mean epoch values. Due to different inclinations in the Cross-Track Pendulum, the nodal drifts will result in different values even if all other orbital parameters have always the same value. Therefore, the ascending nodes of two orbits will separate by

$$
\Delta(\Delta \boldsymbol{\Omega})=\left[\boldsymbol{\Omega}_{2}\left(t_{2}\right)-\boldsymbol{\Omega}_{1}\left(t_{2}\right)\right]-\left[\boldsymbol{\Omega}_{2}\left(t_{1}\right)-\boldsymbol{\Omega}_{1}\left(t_{1}\right)\right]
$$

If this value will exceed a tolerance value $\pm\left(\Delta \lambda_{\Omega}\right)$, a manoeuvre has to be performed in order to reduce the error deviation. A maximum performance in nodal shift will be performed at the northern or southern turn of the orbit, i.e. if the argument of latitude has the value of $90^{\circ}$ or $270^{\circ}$. In this case, the manoeuvre by the angle $\gamma$ will produce the required maximum nodal shift $\Delta \Omega_{\max }$ defined by

$$
\begin{aligned}
& \cos \left(\Delta \boldsymbol{\Omega}_{\max }\right) \sin \left(i_{2}\right)=\cos (\gamma) \sin \left(i_{1}\right) \\
& \sin \left(\Delta \boldsymbol{\Omega}_{\max }\right) \sin \left(i_{2}\right)=\sin (\gamma) \\
& \cos \left(i_{2}\right)=\cos (\gamma) \cos \left(i_{1}\right)
\end{aligned}
$$

Here, $i_{1}$ is the inclination of the satellite orbit before manoeuvre and $i_{2}$ after the manoeuvre. Therefore, a node manoeuvre will also cause the need for an inclination manoeuvre. For a given nodal shift $\Delta \Omega$ and initial inclination $i_{1}$, the last formula allows to calculate the correction angle $\gamma$ and the inclination $i_{2}$ at the end of the correction cycle. Then the inclination $\Delta i=i_{2}-i_{1}$ is also known. The cycle period $T_{\Omega}$ will be calculated by

$T_{\boldsymbol{\Omega}}=\frac{2 \Delta \boldsymbol{\Omega}}{\left|\dot{\boldsymbol{\Omega}}_{S 2}-\dot{\boldsymbol{\Omega}}_{S 1}\right|}$
For the node correction, the corresponding velocity increment will be in the order of

$$
\Delta V_{\Omega}=V_{C} \sqrt{2} \sqrt{1-\cos (\gamma)}=2 V_{C} \sin \left(\frac{\gamma}{2}\right)
$$

and the inclination correction by the inclination increment $\Delta i$ requires

$\Delta V_{i}=V_{C} \sqrt{2} \sqrt{1-\cos (\Delta i)}=2 V_{C} \sin \left(\frac{\Delta i}{2}\right)$

For small angles, these formulae will be reduced to

$\Delta V_{\boldsymbol{\Omega}} \approx V_{C} \gamma$ and $\Delta \boldsymbol{\Omega}_{\max } \approx \frac{\gamma}{\sin (i)}$ and $\Delta V_{i} \approx V_{C} \Delta i$

This second independent manoeuvre in order to correct the inclination has to be performed during the nodal crossing. If $\Omega_{01}$ is the desired node of the orbit, during the mission as initial node the value

$\boldsymbol{\Omega}_{02}=\boldsymbol{\Omega}_{01} \pm \Delta \boldsymbol{\Omega}$

will be used. In this equation the sign has to be selected in such a way, that the relative motion of the node is always inside of the interval $\Omega \in\left[\Omega_{01}-\Delta \Omega, \Omega_{01}+\Delta \Omega\right]$. After the correction cycle $T_{\Omega}$ the manoeuvre will be performed in order to move the node to the other end of the nodal interval. For the correction of the inclination a similar procedure might be applied: select half an interval and bring the inclination at the end of the tolerance interval. However, the variation in the inclination is not easy to predict because of the uncertainties in prediction of the true perturbations. Therefore, it is recommended to apply the whole inclination correction at the end of the nodal shift Based on this assumption, the estimation of the fuel consumption will be obtained.

\subsubsection{Perigee manoeuvre}

Assuming a change in the line of apsides without change of any other orbital elements and for given change angle $\Delta \omega$ in argument of perigee, the true anomaly $v_{1}$ of the satellite in orbit 1 to perform the manoeuvre can be calculated from

$$
\begin{aligned}
\cos \left(v_{1}\right) & = \pm \frac{\sin (\Delta \omega)}{\sqrt{2(1-\cos (\Delta \omega))}}= \pm \frac{\sqrt{1+\cos (\Delta \omega)}}{\sqrt{2}} \\
& =\cos \left(\frac{\Delta \omega}{2}\right), \quad \tan \left(v_{1}\right)=\tan \left(\frac{\Delta \omega}{2}\right)
\end{aligned}
$$

The corresponding true anomaly along orbit 2 will be $v_{2}=v_{1}-\Delta \omega$ and $v_{1}=-v_{2}=\Delta \omega / 2$ or $v_{1}=-v_{2}=\Delta \omega / 2+180^{\circ}$. The corresponding orbital radius and radial velocity will be calculated from

$$
\begin{aligned}
& r=\frac{a\left(1-e^{2}\right)}{1+e \cos \left(v_{1}\right)}=\frac{a\left(1-e^{2}\right)}{1+e \cos \left(v_{2}\right)} \\
& \dot{r}_{i}=e \sqrt{\frac{\mu}{a\left(1-e^{2}\right)}} \sin \left(v_{1}\right) \\
& \dot{r}_{2}=e \sqrt{\frac{\mu}{a\left(1-e^{2}\right)}} \sin \left(v_{2}\right)
\end{aligned}
$$

The radius and velocity vectors will be computed, using the radial and the transversal direction vectors $\vec{r}_{0}$ and $\vec{q}_{0}$ from

$$
\vec{r}_{1}=\vec{r}_{2}=r \vec{r}_{0}
$$


$\vec{r}_{1}=r \dot{\vec{r}}_{0}+\frac{\sqrt{\mu a_{1}\left(1-e^{2}\right)}}{r} \vec{q}_{0}$

$\vec{r}_{2}=r \dot{r_{0}}+\frac{\sqrt{\mu a_{1}\left(1-e^{2}\right)}}{r} \vec{q}_{0}$

. The velocity increment vector is given by $\Delta \dot{\vec{r}}=$ $\vec{r}_{2}-\vec{r}_{1}$ and the radial variation is calculated from $\dot{r}_{1}=-\dot{r}_{2}$. The rotational angle between the velocity vectors on orbit 1 and orbit 2 will be calculated from

$\cos \left(\gamma_{\omega}\right)=\frac{-\dot{r}_{1}^{2}+\left(\mu a\left(1-e^{2}\right) / r^{2}\right)}{\dot{r}_{1}^{2}+\left(\mu a\left(1-e^{2}\right) / r^{2}\right)}$

Finally the velocity increment will be

$\Delta V_{\omega}=V_{1} \sqrt{2} \sqrt{1-\cos \left(\gamma_{\omega}\right)}=2 V_{1} \sin \left(\frac{\gamma_{\omega}}{2}\right)$

For all these calculations, examples are shown in Fig. 5.

As seen from Fig. 5, the variation due to orbital height is in the order of a few percent for changes of the semi-major axis, and almost negligible for changes in eccentricity and/or changes in the perigee. Of course, for formation analyses these differences have to be taken into account.

\subsection{Orbit maintenance}

The variation in the semi-major axis due to air drag is estimated by

$\dot{a}_{s}=-c_{D} \rho \frac{a^{2}}{\mu} \frac{A}{m} V^{3}$

with $c_{D}$ being the air drag coefficient (here assumed as $c_{D}=2.2$ ), satellite mass $m$, the gravitational constant $\mu$, cross section $A$, and the solar activity depending air density $\rho$ at the height of the satellite. $V$ is the satellite's velocity with respect to the inherent air. This velocity is usually unknown and will be replaced by the satellite velocity with respect to the Earth's centre. The semimajor axis correction manoeuvre and its fuel consumption are calculated according to (20) and (32). As an example, the fuel requirement for keeping a microsatellite at its orbital height for one year is shown in Fig. 6.

An additional need for orbit maintenance manoeuvres will occur in case of deviations $\Delta B_{D}$ in the ballistic coefficient

$B_{D}=c_{D} \frac{A}{m}$

as a consequence of different atmosphere flowing towards the different satellites. Based on the orbital decay $\dot{a}_{s}$, the secular change in semi-major axis during the time interval $\Delta t$ follows from $\Delta a=\dot{a}_{s} \Delta \mathrm{t}$. Assuming a deviation $\Delta B_{D}$ in the ballistic coefficients of 2 satellites of the formation system and allowing the variation $\Delta a$ in semi-major axis, a

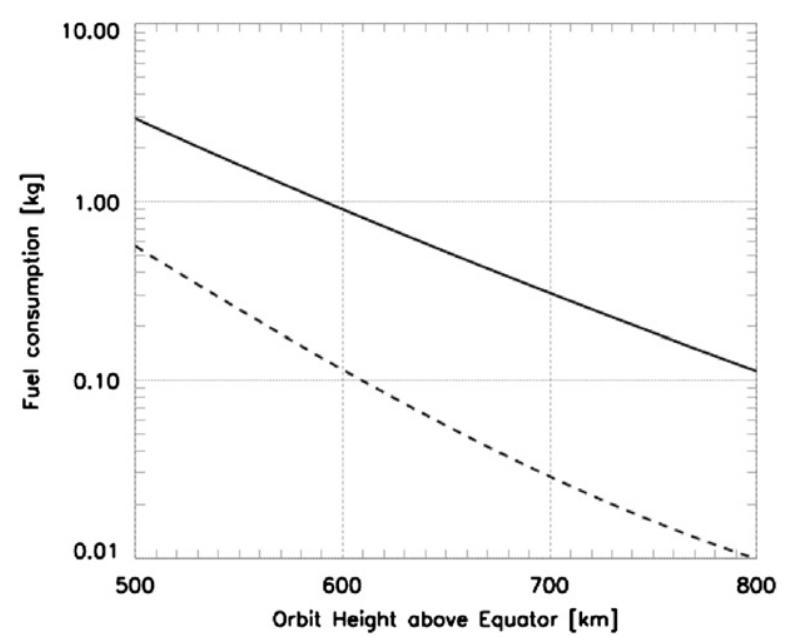

Fig. 6. Fuel consumption for orbit keeping for one year operation time vs. orbit height above the equator for a microsatellite with $130 \mathrm{~kg}$ and a cross section of $2 \mathrm{~m}^{2}$ The solid line represents solar maximum activity and the dashed line solar minimum activity. (Some rough values for the air density $\rho\left[\mathrm{g} / \mathrm{km}^{3}\right]$ from [2]: $H=500 \mathrm{~km}: \rho_{\min }=0.3916 ; \rho_{\max }=2.042$, $H=650 \mathrm{~km}: \rho_{\min }=0.0342, \rho_{\max }=0.3700, H=800 \mathrm{~km}: \rho_{\min }=0.007069$, $\rho_{\max }=0.08059$ ).
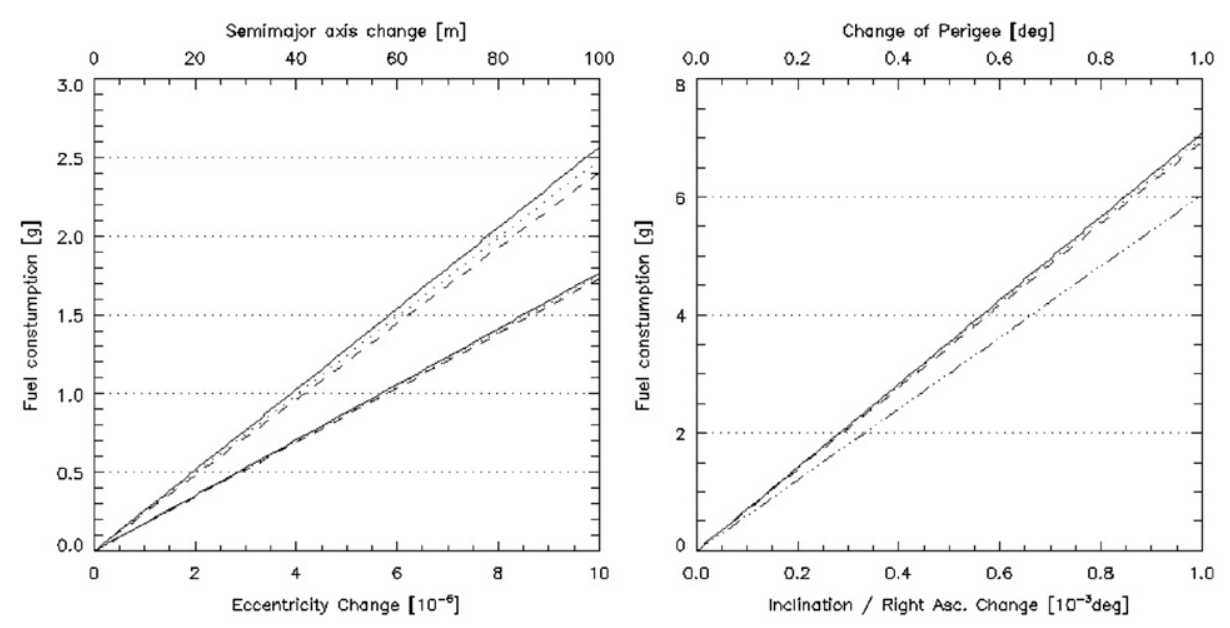

Fig. 5. Left plot: fuel consumption for a change in the semi-major axis (upper abscissa and upper lines: solid (500 km), dotted (650 km), and dashed $(800 \mathrm{~km}))$ and for a change in the eccentricity (lower abscissa and lower lines: solid (500 km), dotted (650 km), and dashed (800 km)). Right plot: fuel consumption for a change in perigee (upper abscissa and upper lines: solid (500 km), dotted (650 km), and dashed (800 km)) and for inclination or right ascension of the ascending node (dotted-dashed line, lower abscissa). The satellite's mass is assumed to be $130 \mathrm{~kg}$. 
relative along track error interval $\Delta L[13]$

$\Delta L=\frac{3(\Delta a)^{2}}{4 \rho a^{2} \Delta B_{D}}$

has to be taken into account. On the other hand, if $\Delta L$ is the allowed tolerance interval for an along track error, the corresponding allowed tolerance deviation $\Delta a$ in semimajor axis follows from

$\Delta a=\sqrt{\Delta L \frac{4 \rho a^{2} \Delta B_{D}}{3}}$

Assuming a circular satellite motion, this formula allows the estimation of fuel requirement for the orbit manoeuvre in order to keep the orbital behaviour of the satellite within the allowed tolerance $\Delta L$. The cycle time $T_{\text {along }}$ for the manoeuvre cycle is given by [13]

$T_{\text {along }}=\frac{4}{\text { an }} \sqrt{\frac{\Delta L}{3 \rho \Delta B_{D}}}=4 \sqrt{\frac{a}{\mu} \frac{\Delta L}{3 \rho \Delta B_{D}}}$

An estimation for the allowed variation in the ballistic coefficient follows for an allowed variation in semi-major axis $\Delta a$ and the desired cycle time $T_{\text {along }}$ from

$\Delta B_{D}=\frac{2 \Delta a}{\rho T_{\text {along }} \sqrt{\mu a}}$

\subsection{Formation maintenance}

To maintain the different formations, individual manoeuvres must be applied as depicted in Table 2. The respective manoeuvres follow from the formulae given in Sections 4.2. and 4.3.

\subsection{Distance maintenance}

The distance between the formation and the master satellite will vary due to different ballistic coefficients. This will be adjusted by semi-major axis manoeuvres as described in Section 4.3 and will be performed with the orbit maintenance manoeuvres.

\section{Collision avoidance strategy}

If a small satellite does not function it might be desired to separate satellites for a certain time span and rebuild the formation after some days. Therefore, the orbit position separation based on anomalistic motion as described in Section 4.1.1 is a low-cost solution with respect to fuel consumption. Here, the satellites are injected into elliptic orbits, which separate quite quickly each satellite from another. These orbits are chosen such that the satellites will be at the same position after some pre-defined time or, if desired, the satellites are separated by a certain distance. The mal-functioning satellite might then be analysed, and the formation reconfigured if the erroneous behaviour is found. As an example for the TerraSAR-X as master satellite, which is the most sensitive case, the satellites shall be separated by $50 \mathrm{~km}$ with two manoeuvres with this strategy. The first satellite should perform $N=60$ anomalistic periods and the second satellite $M=61$ anomalistic periods. The second satellite will be injected into an orbit with $a=6878.218 \mathrm{~km}$ with an eccentricity of $e=0.007692$ applying the velocity increment of $\Delta V=-5.855 \mathrm{~m} / \mathrm{s}$. The total transfer will last $95.479 \mathrm{~h}$ and requires a fuel mass of $\Delta m=0.272 \mathrm{~kg}$. Now, the satellites are separated and can be brought together with the same amount of fuel within approx. four days.

\section{Results}

To gain a feeling for fuel consumption for the different formations at different heights, the fuel requirements for five exemplary formations are calculated: The Cartwheel and some different Pendulum formations, e.g. the Trinodal Pendulum*. The latter will hold a small eccentricity offset corresponding to a vertical separation at the northern/ southern turns of $50 \mathrm{~m}$. This separation will be kept constant by means of orbital manoeuvres. The Cartwheel will have a wheel size of $5 \mathrm{~km}$, that means a $\Delta e$ will be $2500 \mathrm{~m}$ for a single satellite. For comparison, the Trinodal Pendulum* will consist of a separation of $5 \mathrm{~km}$ at the equator of two satellites with the third satellite being placed in the middle. Here, these $5 \mathrm{~km}$ are seen more as an example to compare both formations-radar constraints and interferometric applications may change this value to different values. The formations are assumed to orbit at 500,650 , and $800 \mathrm{~km}$ to point out the effects of atmospheric drag. In each case an average distance between the small satellites formations reference point and the master of $50 \mathrm{~km}$ will be assumed. The formations shall be kept stable for a total lifetime of 2 years and are assumed to be operated during solar maximum activity. Of course, in the calculations the formation acquisition is included, assuming that the microsatellites are launched together with the illuminating spacecraft. The results are summarized in the following Tables.

A deviation of the ballistic coefficient between the satellites within a formation is assumed to be $1 \%$. The nodal crossing reference longitude was assumed to be within a margin of $\pm 1 \mathrm{~km}$.

The results should be compared in a qualitative sense, not in the last detail in a quantitative sense.

The single formations numerically investigated are kept and maintained with the following restrictions:

Cartwheel formation acquisition (see Fig. 7, left picture): correction of semi-major axis in order to maintain the mean draconic period $(H=500 \mathrm{~km}: \Delta a=0.004 \mathrm{~km} ; H=650 \mathrm{~km}$ and $H=800 \mathrm{~km}: \Delta a=0.002 \mathrm{~km})$, separation of satellite relative position by $\pm 5.3 \mathrm{~km}$, argument of perigee manoeuvre in order to get a relative separation of the apsidal lines by $\pm 120^{\circ}$.

Cartwheel formation maintenance: It will be assumed that formation maintenance manoeuvres will be required by a relative difference on the ballistic coefficient of $10 \%$ induced by small differences in the influence of the air drag on the three satellites. In addition, an along track error of $100 \mathrm{~m}$ shall be tolerated. If in addition an inertial stabilization with respect to the long-term motion of the apsidal lines is required, an additional fuel amount in the range of up to about $6 \mathrm{~kg}$ for the 2 years lifetime will be required for each of the microsatellites [4] (Table 3 ). 

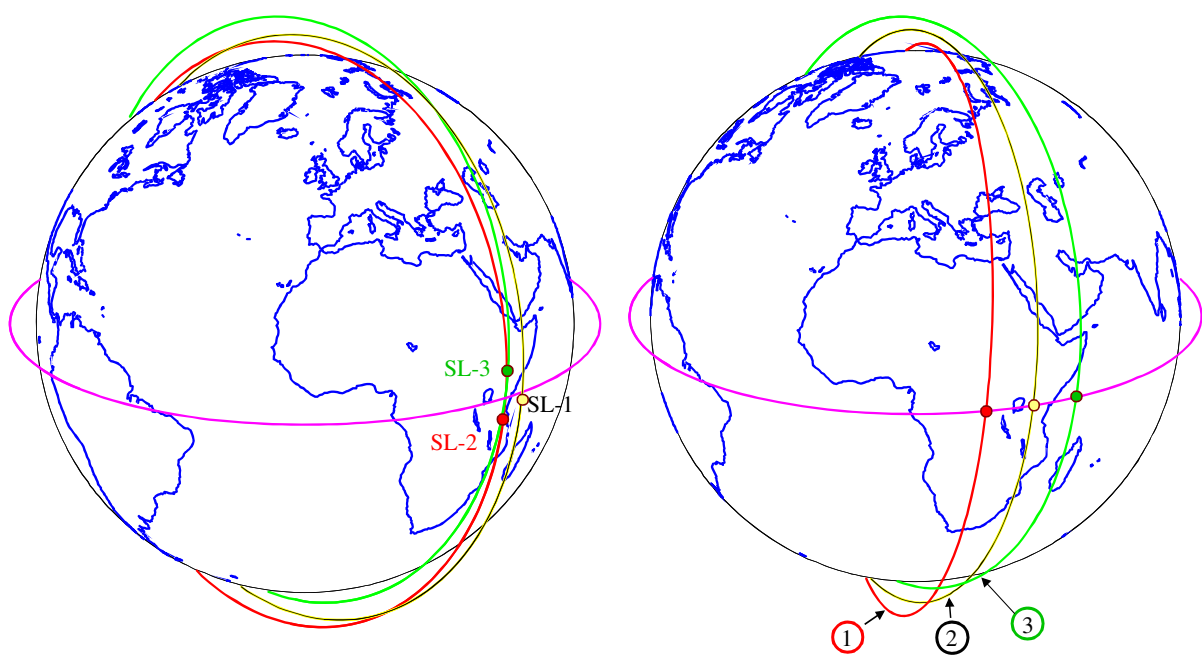

Fig. 7. Schematic representation of the Cartwheel configuration (left figure) and the Trinodal Pendulum* configuration (right figure).

Table 3

Total fuel requirement in [kg] for the Cartwheel formations including orbit keeping, formation keeping, and all maintenance manoeuvres for 2 years for solar maximum activity. Decrease in drag becomes apparent in the fuel requirement for higher orbital altitudes (orbit maintenance). The same effect is visible in the other formations.

\begin{tabular}{|c|c|c|c|c|c|c|}
\hline Cartwheel & $\begin{array}{l}\text { Formation } \\
\text { satellite }\end{array}$ & $\begin{array}{l}\text { Orbit } \\
\text { acquisition }\end{array}$ & $\begin{array}{l}\text { Orbit } \\
\text { maintenance }\end{array}$ & $\begin{array}{l}\text { Formation } \\
\text { acquisition }\end{array}$ & $\begin{array}{l}\text { Formation } \\
\text { maintenance }\end{array}$ & $\begin{array}{l}\text { Total fuel } \\
\text { requirement }\end{array}$ \\
\hline \multirow[t]{3}{*}{$500 \mathrm{~km}$} & Sat1 & 0.0045 & 5.762 & 0.006423 & 0.587 & 6.360 \\
\hline & Sat2 & 0.0045 & 5.762 & 0.029474 & 0.587 & 6.383 \\
\hline & Sat3 & 0.0045 & 5.762 & 0.029484 & 0.587 & 6.383 \\
\hline \multirow[t]{3}{*}{$650 \mathrm{~km}$} & Sat1 & 0.0045 & 0.609 & 0.006219 & 0.116 & 0.736 \\
\hline & Sat2 & 0.0045 & 0.609 & 0.029041 & 0.116 & 0.759 \\
\hline & Sat3 & 0.0045 & 0.609 & 0.029054 & 0.116 & 0.759 \\
\hline \multirow[t]{3}{*}{$800 \mathrm{~km}$} & Sat1 & 0.0044 & 0.270 & 0.006025 & 0.022 & 0.302 \\
\hline & Sat2 & 0.0044 & 0.270 & 0.027649 & 0.022 & 0.324 \\
\hline & Sat3 & 0.0044 & 0.270 & 0.027650 & 0.022 & 0.324 \\
\hline
\end{tabular}

Cross-track Pendulum formation acquisition: Microsatellite 1 will get the (sunsynchronous) injection inclination, microsatellite 2 a deviation by about $\Delta i=0.018^{\circ}$ for all altitudes, microsatellite 3 a deviation by about $\Delta i=-0.018^{\circ}$ for all altitudes. Accordingly the draconic period of satellites 2 and 3 will be modified with respect to the master satellite. This can be balanced by increasing the semi-major axis, in case of the $H=500 \mathrm{~km}$ orbit by the value $\Delta a=6 \mathrm{~m}$. This small value will not be respected during formation acquisition. The orbital planes of the other two satellites are separated by a small shift in right ascension of the ascending node by $\Delta \Omega= \pm 0.021^{\circ}$.

Cross-track Pendulum formation maintenance: Similar to the Cartwheel considerations an error in the ballistic coefficient of about $10 \%$ will be taken into account. Therefore similar values for the fuel amount during the two years satellite lifetime have to be expected. However, for microsatellite 1 due to the modified inclination additional manoeuvres have to be foreseen. Due to the very small orbital eccentricities no variation in the apsidal lines is investigated in this context (Table 4).
CarPe formation acquisition: Similar to the procedure of the Cross-track Pendulum Formation the orbital planes of two microsatellites are separated by a small shift in right ascension of the ascending node by $\Delta \Omega= \pm 0.021^{\circ}$. However the other satellite (in the example microsatellite 3) will get a modified eccentricity (not an inclination change as in the previous case) in order to minimize a collision risk and allow interferometric observation.

CarPe formation maintenance: Similarly to the Cartwheel considerations an error in the ballistic coefficient of about $10 \%$ will be taken into account. Therefore similar values for the fuel amount during the two years satellite lifetime has to be expected. However for microsatellite 3 due to the modified eccentricity additional very small manoeuvres have to be foreseen (Table 5).

Trinodal Pendulum acquisition: The first of the three microsatellites is assumed to fly on an orbit with identical semi-major axis, eccentricity and inclination as the master satellite. The other two microsatellites move on orbital planes separated by a change in the right ascension of ascending node (example for $H=500 \mathrm{~km}$ orbit: $\Delta \Omega_{1}=0.021^{\circ}, \Delta \Omega_{2}=-0.021^{\circ}$ ), whereas all other orbital 
Table 4

Total fuel requirement in $[\mathrm{kg}]$ for the Cross-track Pendulum formations including orbit keeping, formation keeping, and all maintenance manoeuvres for 2 years for solar maximum activity.

\begin{tabular}{|c|c|c|c|c|c|c|}
\hline $\begin{array}{l}\text { Cross-track } \\
\text { Pendulum }\end{array}$ & $\begin{array}{l}\text { Formation } \\
\text { satellite }\end{array}$ & $\begin{array}{l}\text { Orbit } \\
\text { acquisition }\end{array}$ & $\begin{array}{l}\text { Orbit } \\
\text { maintenance }\end{array}$ & $\begin{array}{l}\text { Formation } \\
\text { acquisition }\end{array}$ & $\begin{array}{l}\text { Formation } \\
\text { maintenance }\end{array}$ & $\begin{array}{l}\text { Total fuel } \\
\text { requirement }\end{array}$ \\
\hline \multirow[t]{3}{*}{$500 \mathrm{~km}$} & Sat1 & 0.0045 & 5.762 & 0.0 & 5.307 & 11.074 \\
\hline & Sat2 & 0.0045 & 5.762 & 0.349 & 5.894 & 12.010 \\
\hline & Sat3 & 0.0045 & 5.762 & 0.349 & 5.894 & 12.010 \\
\hline \multirow[t]{3}{*}{$650 \mathrm{~km}$} & Sat1 & 0.0045 & 0.609 & 0.0 & 4.856 & 5.470 \\
\hline & Sat2 & 0.0045 & 0.609 & 0.438 & 4.972 & 6.024 \\
\hline & Sat3 & 0.0045 & 0.609 & 0.438 & 4.972 & 6.024 \\
\hline \multirow[t]{3}{*}{800 km } & Sat1 & 0.0044 & 0.270 & 0.0 & 4.449 & 4.723 \\
\hline & Sat2 & 0.0044 & 0.270 & 0.335 & 4.474 & 5.083 \\
\hline & Sat3 & 0.0044 & 0.270 & 0.335 & 4.474 & 5.083 \\
\hline
\end{tabular}

Table 5

Total fuel requirement in $[\mathrm{kg}]$ for the CarPe formations including orbit keeping, formation keeping, and all maintenance manoeuvres for 2 years for solar maximum activity.

\begin{tabular}{|c|c|c|c|c|c|c|}
\hline CarPe & $\begin{array}{l}\text { Formation } \\
\text { satellite }\end{array}$ & $\begin{array}{l}\text { Orbit } \\
\text { acquisition }\end{array}$ & $\begin{array}{l}\text { Orbit } \\
\text { maintenance }\end{array}$ & $\begin{array}{l}\text { Formation } \\
\text { acquisition }\end{array}$ & $\begin{array}{l}\text { Formation } \\
\text { maintenance }\end{array}$ & $\begin{array}{l}\text { Total fuel } \\
\text { requirement }\end{array}$ \\
\hline \multirow[t]{3}{*}{$500 \mathrm{~km}$} & Sat1 & 0.0045 & 5.762 & 0.124 & 0.587 & 6.478 \\
\hline & Sat2 & 0.0045 & 5.762 & 0.124 & 0.587 & 6.478 \\
\hline & Sat3 & 0.0045 & 5.762 & 0.016 & 0.593 & 6.378 \\
\hline \multirow[t]{3}{*}{$650 \mathrm{~km}$} & Sat1 & 0.0045 & 0.609 & 0.123 & 0.116 & 0.853 \\
\hline & Sat2 & 0.0045 & 0.609 & 0.123 & 0.116 & 0.853 \\
\hline & Sat3 & 0.0045 & 0.609 & 0.015 & 0.117 & 0.745 \\
\hline \multirow[t]{3}{*}{800 km } & Sat1 & 0.0044 & 0.270 & 0.122 & 0.022 & 0.418 \\
\hline & Sat2 & 0.0044 & 0.270 & 0.122 & 0.022 & 0.418 \\
\hline & Sat3 & 0.0044 & 0.270 & 0.015 & 0.022 & 0.311 \\
\hline
\end{tabular}

Table 6

Total fuel requirement in $[\mathrm{kg}]$ for the Trinodal Pendulum formations including orbit keeping, formation keeping, and all maintenance manoeuvres for 2 years for solar maximum activity.

\begin{tabular}{llllll}
\hline $\begin{array}{l}\text { Trinodal } \\
\text { Pendulum }\end{array}$ & $\begin{array}{l}\text { Formation } \\
\text { satellite }\end{array}$ & $\begin{array}{l}\text { Orbit } \\
\text { acquisition }\end{array}$ & $\begin{array}{l}\text { Orbit } \\
\text { maintenance }\end{array}$ & $\begin{array}{l}\text { Formation } \\
\text { acquisition }\end{array}$ & $\begin{array}{l}\text { Formation } \\
\text { maintenance }\end{array}$ \\
\hline $\mathbf{5 0 0} \mathbf{~ k m}$ & Sat1 & 0.0045 & 5.762 & 0.128 & 0.587 \\
& Sat2 & 0.0045 & 5.762 & 0.000 & 0.587 \\
& Sat3 & 0.0045 & 5.762 & 0.128 & 0.587 \\
$\mathbf{6 5 0} \mathbf{~ k m}$ & Sat1 & 0.0045 & 0.609 & 0.125 & 0.117 \\
& Sat2 & 0.0045 & 0.609 & 0.000 & 0.117 \\
$\mathbf{8 0 0} \mathbf{~ k m}$ & Sat3 & 0.0045 & 0.609 & 0.125 & 0.117 \\
& Sat1 & 0.0044 & 0.270 & 0.121 & 0.754 \\
& Sat2 & 0.0044 & 0.270 & 0.000 & 0.022 \\
& Sat3 & 0.0044 & 0.270 & 0.121 & 0.025 \\
\end{tabular}

Table 7

Total fuel requirement for the Trinodal Pendulum* formations including orbit keeping, formation keeping, and all maintenance manoeuvres for 2 years for solar maximum activity. The vertical separation at the northern/southern turns is assumed to be $50 \mathrm{~m}$.

\begin{tabular}{llllll}
\hline $\begin{array}{l}\text { Trinodal } \\
\text { Pendulum* }\end{array}$ & $\begin{array}{l}\text { Formation } \\
\text { satellite }\end{array}$ & $\begin{array}{l}\text { Orbit } \\
\text { acquisition }\end{array}$ & $\begin{array}{l}\text { Orbit } \\
\text { maintenance }\end{array}$ & $\begin{array}{l}\text { Formation } \\
\text { acquisition }\end{array}$ & $\begin{array}{l}\text { Formation } \\
\text { maintenance }\end{array}$ \\
\hline $\mathbf{5 0 0} \mathbf{~ k m}$ & Sat1 & 0.0045 & 5.762 & 0.128 & 0.638 \\
& Sat2 & 0.0045 & 5.762 & 0.000 & 0.587 \\
& Sat3 & 0.0045 & 5.762 & 0.128 & 0.638 \\
$\mathbf{6 5 0} \mathbf{~ k m}$ & Sat1 & 0.0045 & 0.609 & 0.125 & 0.168 \\
& Sat2 & 0.0045 & 0.609 & 0.000 & 0.117 \\
$\mathbf{8 0 0} \mathbf{~ k m}$ & Sat3 & 0.0045 & 0.609 & 0.125 & 0.168 \\
& Sat1 & 0.0044 & 0.270 & 0.121 & 0.907 \\
& Sat2 & 0.0044 & 0.270 & 0.000 & 0.073 \\
& Sat3 & 0.0044 & 0.270 & 0.121 & 0.023 \\
\end{tabular}


elements are unchanged. By this way, no deviation in the draconic period must be taken into account.

Trinodal Pendulum maintenance: Similarly to the Cartwheel considerations an error in the ballistic coefficient of about $10 \%$ will be taken into account. Therefore similar values for the fuel amount during the two years satellite lifetime have to be expected (Table 6).

Trinodal Pendulum* acquisition (see Fig. 7, right picture): The first of the three microsatellites is assumed to fly on an orbit with identical semi-major axis, eccentricity, and inclination as the master satellite. The other two microsatellites move on orbital planes separated by a change in the right ascension of ascending node (example for $H=500 \mathrm{~km}$ orbit: $\Delta \Omega_{2}=0.021^{\circ}, \Delta \Omega_{3}=-0.021^{\circ}$ ). In addition these two satellites will get a change in eccentricity (example for $H=500 \mathrm{~km}$ orbit: $e_{2}=e_{3}=0.000036$ )

Trinodal Pendulum* maintenance: As in the Cartwheel considerations, an error in the ballistic coefficient of about $10 \%$ will be taken into account. Therefore similar values for fuel consumption during the two years satellite lifetime have to be expected. The change in eccentricity causes a small change in draconic period. This is considered by assuming a small change in the error of the ballistic coefficient by $10.1 \%$. Similarly, a small change in the secular drift of the right ascension of the ascending node (cf. formula (34)) has to be taken into account. This value varies the fuel amount for formation acquisition. However, this value is so small that it cannot be reflected in Table 7. But during formation control this effect will be included among other higher-order perturbations not considered here. All these additional perturbations have a negligible impact on the qualitative fuel estimations in this paper.

Note: In all examples the fuel amount for orbit maintenance is represented by its highest value. According

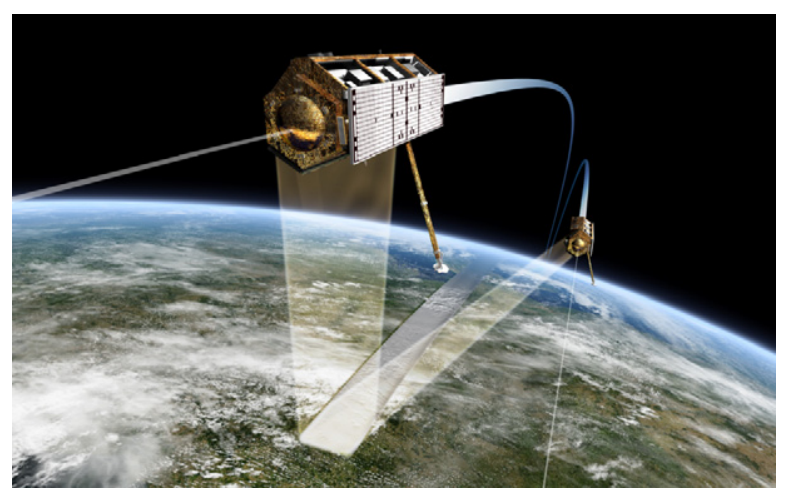

Fig. 8. Artists view of the formation flight of TerraSAR-X and TanDEM-X on a HELIX orbit. to Fig. 6 the amount for the low $500 \mathrm{~km}$ orbit is essentially higher than for the upper orbits. This is caused by the strong air drag in this low altitude and therefore an essential design driver for selection of a suitable constellation flight.

\section{Discussion}

Assuming an orbit injection of the small satellites together with the master satellite, the present study considers fuel consumption for the orbit positioning of the small satellites in an average distance of $50 \mathrm{~km}$ with respect to the master satellite (orbit acquisition), the orbit maintenance, the formation acquisition, and the formation maintenance, each for a two years time span. Independent of the formation, in any case the orbit maintenance is the most demanding factor for fuel need during the satellites planned lifetime. Formation acquisition and formation maintenance require more or less the identical fuel amount for any constellation. It can be seen that the fuel demand depends on orbit height, which is induced by drag and driven by solar activity. For a lifetime of two years, fuel consumption is not the limiting factor for any formation. Therefore, for a satellite formation design other constraints shall be taken into account like required distances between the satellites (depending on requested applications), which might require, e.g. autonomous control, allowing for a smaller formation at a lower orbital height. Other constraints might be, e.g. in case of SAR interferometry, overlapping Doppler spectra. Here, the along track distance between two data recording satellites should be as small as possible, leading to formations with small or constant along track distances like, e.g., the Trinodal Pendulum. Only in case of a very long lasting satellite operation with small satellites, orbit height might be a design driver to reduce fuel consumption.

Another constraint for satellites flying in close formation is avoiding any collision. Here, autonomous control with proper control manoeuvres might reduce this possibility. Another strategy might be an implementation of the proposed separation manoeuvres, which might be executed if, e.g., the distance between two satellites is smaller than a given threshold. The distance might be measured by GPS onboard each satellite and its signals might then be exchanged via an inter-satellite link. Three different methods are proposed, which separate one satellite from the formation and reunite it after a given time. The fuel requirement is the same for all methods while the number of manoeuvres is changing from 2 to 4 , leaving it to the user to choose the best for his purpose.

Table 8

Orbital data for the TerraSAR-X (TSX)/TanDEM-X (TDX) formation flight on a HELIX orbit. The values $A_{2}$ and $B_{2}$ represent snapshots of the formation and are being adjusted during the mission according to the required geometry.

\begin{tabular}{|c|c|c|c|c|c|c|}
\hline TSX & $A_{1}=0 \mathrm{~m}$ & $\Delta e_{1}=0.0$ & $\alpha_{1}=0^{\circ}$ & $M_{M 0}=-90^{\circ}$ & $\Delta M_{1}=180^{\circ}$ & $B_{1}=0 \mathrm{~m}$ \\
\hline TDX & $A_{2}=300 \mathrm{~m}$ & $\Delta e_{2}=0.000044$ & $\alpha_{2}=180^{\circ}$ & $\omega_{M}=-90^{\circ}$ & $\Delta M_{2}=0^{\circ}$ & $B_{2}=400 \mathrm{~m}$ \\
\hline TSX & $\beta_{1}=90^{\circ}$ & $u_{M 0}=0^{\circ}$ & $\Delta i_{1}=0^{\circ}$ & $\Delta \Omega_{1}=0^{\circ}$ & $\Delta y_{1}=0 \mathrm{~m}$ & $\Delta \omega_{1}=0^{\circ}$ \\
\hline TDX & $\beta_{2}=90^{\circ}$ & & $\Delta i_{2}=0^{\circ}$ & $\Delta \Omega_{1}=0^{\circ} .00335^{\circ}$ & $\Delta y_{2}=0 \mathrm{~m}$ & $\Delta \omega_{2}=0.0004^{\circ}$ \\
\hline
\end{tabular}


An example for the technical realisation of a formation flight as an application of the formulae presented in this paper is the TanDEM-X mission $[15,16,18]$. This mission employs two formation flying radar satellites to acquire a global digital elevation model (DEM) with unprecedented accuracy. The first satellite (TerraSAR-X) was launched on June 15, 2007 into an 11 days sunsynchronous duskdawn repeat orbit with a semi-major axis $a_{M}=6883.5 \mathrm{~km}$ (actual data, corresponding to a mean orbital height of $505 \mathrm{~km}$ ) and an inclination $i_{M}=97.444^{\circ}$. The second satellite followed on June 21, 2010, and has been successfully injected into a HELIX orbit with respect to TerraSAR-X. The orbits are visualized in Fig. 7 on the right: TerraSAR-X can be assumed as satellite 2 , whereas the second TanDEM-X satellite either as satellite 1 or satellite 3 , respectively. An artist's visualisation of the formation flight of the two satellites together with the observation scheme is shown in Fig. 8. According to Table 1 the data summarized in Table 8 are chosen in order to select a suitable HELIX orbit. The parameter values $A_{2}$ and $B_{2}$ will slightly be adjusted during the three years mission time in order to optimize the DEM performance on a global scale.

\section{References}

[1] D. Brouwer, Solution of the problem of artificial satellite theory without drag, Astronomical Journal 64 (1959) 378-397.

[2] J.O. Capellari, C.E. Velez, A.J. Fuchs, Mathematical Theory of the Goddard Trajectory Determination System, GSFC, Greenbelt, Maryland, 1976.

[3] W.H. Clohessy, R.S. Wiltshire, Terminal guidance systems for satellite rendezvous, Journal of Aerospace Sciences 27 (9) (1960) 653-674.

[4] S. D'Amico, Private communication 2007.

[5] A. Das, R. Cobb, M. Stallard, TechSat 21: A revolutionary concept in distributed space based sensing, in: Proceedings of the AIAA Defense and Civil Space Programs Conference and Exhibit, AIAA 98-5255, 1998.

[6] W. Enderle, H. Fiedler, S. De Florio, G. Krieger, F. Jochim, S. D’Amico, S. Dawson, W. Kellar, Next generation GNSS for navigation of future SAR constellations, International Astronautical Congress, 2006; $\langle$ http://elib.dlr.de/45108 >.

[7] N.B. Evans, P. Lee, R. Girard, The Radarsat $2 \& 3$ topographic mission: an overview, in: Proceedings IGARSS02, Toronto, Canada, 22-26 June, 2002.
[8] H. Fiedler, G. Krieger, Close formation flight of passive receiving micro-satellites, in: Proceedings of the 18th International Symposium on Space Flight Dynamics, Munich, 11-15 October, 2004 pp. 1-6.

[9] G.W. Hill, Researches in lunar theory, American Journal of Mathematics, Pure and Applied 1 (1878) 5-26.

[10] E.F. Jochim, Orbit aspects of TS-L/CTW formation flight, GSOC Technical Note TN 2003-06, October 28, 2003.

[11] E.F. Jochim, M. Kirschner, H. Fiedler, Estimation of fuel requirement for Bi-static orbit configurations, DLR GSOC, Technical Note TN 2004-03, 2005.

[12] E.F. Jochim, M. Kirschner, H. Fiedler, G. Krieger, Suitable orbits for simultaneous SAR interferometry, DLR GSOC Technical Note TN 2003-04, 2003.

[13] M. Kirschner, O. Montenbruck, S. Bettapur, Flight dynamics aspects of the Grace formation flying, in: Proceedings of the Space Flight Dynamics Conference, Toulouse, 2001, p. 187.

[14] G. Krieger, H. Fiedler, J. Mittermayer, K. Papathanassiou, A. Moreira, Analysis of multistatic configurations for spaceborne SAR interferometry, IEE Proceedings Radar, Sonar, Navigation 150 (No. 3) (2003) 87-96.

[15] G. Krieger, A. Moreira, H. Fiedler, I. Hajnsek, M. Zink, M. Werner, M Eineder, TanDEM-X: mission concept, product definition and performance prediction, in: Proceedings of the European Conference on Synthetic Aperture Radar (EUSAR), VDE Verlag GmbH, Dresden, Germany, 2006, pp. 1-4.

[16] G. Krieger, A. Moreira, H. Fiedler, I. Hajnsek, M. Werner, M. Younis M. Zink, TanDEM-X: a satellite formation for high resolution SAR interferometry, IEEE Transactions on Geoscience and Remote Sensing 45 (No. 11) (2007) 3317-3341

[17] D. Massonnet, Capabilities and limitations of the interferometric cartwheel, IEEE Transaction on Geoscience Remote Sensing 39 (No. 3) (2001) 506-520.

[18] A. Moreira, et al., TanDEM-X: a TerraSAR-X add-on satellite for single-pass SAR interferometry, IGARSS 2004, Anchorage, USA 2004

[19] H. Runge, R. Bamler, J. Mittermayer, E.F. Jochim, D. Massonnet, E. Thouvenot, The interferometric cartwheel for Envisat, in Proceedings of the Third IAA Symposium on Small Satellites for Earth Observation, Berlin, Germany, 2001

[20] R.J. Sedwick, E.M.C. Kong, D.W. Miller, Exploiting orbital dynamics and micropropulsion for aperture synthesis using distributed satellite systems: Applications to TechSat 21, AIAA_98_5289, 1998

[21] Angélique Vacaresse, Pascal Brousse, Analyse de mission 'Roue Interférométrique' associée au satellite japonais ALOS, CNES Departement de Mission de Mècanique Spatiale, Division Mathématiques Spatiales, Sema Group, Octobre 2000.

[22] H. Zebker, T. Farr, R. Salazar, T. Dixon, Mapping the world's topography using radar interferometry: the TOPSAT mission, Proceedings of the IEEE 82 (12) (1994) 1774-1786.

[23] M. Zink, G. Krieger, T. Amiot, Interferometric performance of a cartwheel constellation for TerraSAR-L, Fringe Workshop, Frascati, Italy, 2003 